\title{
Dynamic Algorithm Selection for SMT
}

\author{
Nikhil Pimpalkhare \\ University of California, Berkeley
}

\begin{abstract}
We describe an online approach to SMT solver selection using nearest neighbor classification and runtime estimation. We implement and evaluate our approach with MedleySolver, finding that it makes nearly optimal selections and evaluates a dataset of queries three times faster than any indivdual solver.
\end{abstract}

ACM Reference Format:

Nikhil Pimpalkhare. 2020. Dynamic Algorithm Selection for SMT. In 35th IEEE/ACM International Conference on Automated Software Engineering (ASE '20), September 21-25, 2020, Virtual Event, Australia. ACM, New York, NY, USA, 3 pages. https://doi.org/10.1145/3324884.3418922

\section{INTRODUCTION}

Satisfiability Modulo Theories (SMT) is an wide field of constraint satisfaction problems over first order theories with many applications, including system verification and automated software testing. There are many general-purpose SMT solvers, such as Z3 [4], CVC4 [3], Yices [5], and Boolector [8]. The decision problems involved are NP-Hard, so each solver uses unique heuristics and tactics, leading to performance differentiation on different queries.

Recent work has shown that machine learning techniques can be used to solve SMT queries faster. For example, FastSMT [2] is a tool which uses neural networks to learn an optimal sequence of tactics, or query transformations, for SMT solvers to use. Problematically, the complexity of such learning methods leads to training times grossly larger than the time spent solving. When evaluating FastSMT, we found that roughly three billion queries would have to be evaluated before the time spent training was justified by the ensuing speedup. Because of the large differences between SMT domains, learning algorithms must re-train for each dataset, so this costly training would be required for each new use case.

Our critical observation is that when a learning algorithm is optimizing for time, training duration must be considered. With this in mind, we chose to approach the problem of algorithm selection for SMT solvers in an online manner. In doing so, we ensure that the cost of our training remains small, proportional, and justified.

Our contributions are as follows: (1) A novel nearest neighbor-based approach for solver selection, in which similar past queries are used to make decisions about future ones. (2) A novel approach to predicting when a given solver will timeout on a query where runtimes are modeled and estimated dynamically as exponential distributions. (3) The MedleySolver Tool, a solver selection tool which takes a dataset of SMT queries as input and attempts to

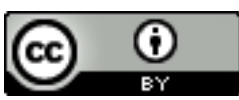

This work is licensed under a Creative Commons Attribution International 4.0 License. ASE '20, September 21-25, 2020, Virtual Event, Australia

(c) 2020 Copyright held by the owner/author(s).

ACM ISBN 978-1-4503-6768-4/20/09.

https://doi.org/10.1145/3324884.3418922 dispatch its predefined pool of solvers optimally. (4) An empirical evaluation of MedleySolver which showing completed evaluation of a bit-vector dataset $3 \mathrm{x}$ faster than the next best individual solver.

\section{RELATED WORK}

FastSMT [2]. FastSMT is a learning tool for speeding up SMT solvers that produces an interpretable learned strategy which can often be significantly faster than a default solver. Due to its complex learning process, FastSMT has an enormous training time which is out of proportion with the number of queries it seeks to solve.

MachSMT [10]. MachSMT is an algorithm selection tool for SMT, making it the most similar to the work presented here. MachSMT employs offline empirical-hardness models to map queries to solvers, and requires a large initial training time.

Multi-Armed Bandit (MAB) [6]. The multi-armed bandit problem is where an agent must select between choices with unknown reward distributions in multiple rounds. The contextual MAB adds access to a feature vector before each round. Solver selection can be viewed as a contextual MAB where the choices are the available solvers and rewards are based on runtime. However, our approach differs in that we select a sequence of solvers for a single query instead of the single solver choice in the MAB formulation.

\section{APPROACH}

Our approach aims to minimize the cumulative time spent processing an inputted set of queries. To accomplish this, for each query, we select an ordering for inputted solvers to run, and attempt to split the pre-allotted runtime per query amongst our solvers in the most advantageous possible way. We process every inputted query in sequence, and learn as we go.

Our first key idea is that problems with similar feature vectors will have similar performance profiles. To leverage this fact, we used a nearest neighbor approach, predicting optimal solvers based on the most similar past queries. For each new query, we sort our list of past solved queries based on L2 norm distance to the current feature vector and extract the $10 \%$ most similar past instances. From this set, we re-sort based on solve speed. We use the order of appearances in this final list-solvers which do not appear are appended to the end of the ordering randomly. Additionally, we select random orderings with decaying probability for initial exploration.

Our second key idea is to find the best way to split the given timeout amongst the solvers. An observable characteristic of SMT solvers is that they often solve queries quickly, or not at all; their runtime resembles an exponential distribution. As such, we terminate a running solver once we are highly confident that it is unlikely to solve the current query.

Specifically, our goal is to find a cutoff timeout value per solver which has a $95 \%$ probability of being larger than that solver's next solve time. To accomplish this, we model each solver's runtime as an exponential distribution. Using Maximum Likelihood Estimation 


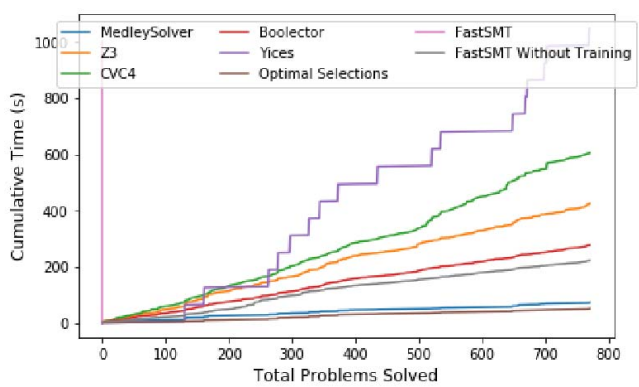

Figure 1: MedleySolver evaluates the given dataset much faster than any other solver, consistently remains very close to the optimal possible runtime, and completes evaluation before tools like FastSMT have finished training.

(MLE) [7], we are able to estimate the most likely parameter for this distribution dynamically using past queries and compute the desired cutoff. For an exponential distribution, the estimation of the single parameter, $\lambda$, is the reciprocal of the mean of the sample points, a value that can be efficiently dynamically updated.

During execution, we proceed through the above ordering using these estimated cutoffs. If we reach the cutoff, we start the next solver. The final solver in our ordering gets the remaining timeout.

Implementation. Our implementation is MedleySolver, available on Github [9]. Featurization uses a Bag of Words encoding and a set of solver-defined metrics, same as FastSMT [2]. This takes insignificant time since the solvers do far more computation after selection. The input is a directory of smt 2 queries, and the output is the result, solver used, and time elapsed per query.

\section{EMPIRICAL EVALUATION}

Models and Methods. We seek to answer four research questions with our evaluation: (1) Does MedleySolver improve upon the inputted solvers? (2) How does Medley Solver compare to offline learning systems when including training time? (3) Is our nearest neighbor approach better than other common approaches to the MAB problem? (4) Does estimating timeout from an exponential distribution improve upon evenly-divided splits?

To answer our research questions, we use graphs in which the $\mathrm{x}$-axis is the number of queries solved thus far, and the $\mathrm{y}$-axis is the cumulative time spent. Unless otherwise specified, all tools are run without any previous training. For consistency, all graphs were generated by evaluating the 2018-Goel-hwbench dataset [11] in the QF-BV (quantifier free bit-vector) theory.

Results. In Fig. 1, we visualize the performance of MedleySolver compared to the four inputted solvers and FastSMT. We did not compare to a pre-trained MachSMT because the authors trained it on our test data, and we were unable to successfully retrain the tool. As shown, MedleySolver is faster than any individual solver, completing the dataset three times faster than the fastest individual solver (Boolector). All solvers complete evaluation long before tools like FastSMT finish training; even ignoring training, MedleySolver is faster than FastSMT. For our first two research questions, Medley

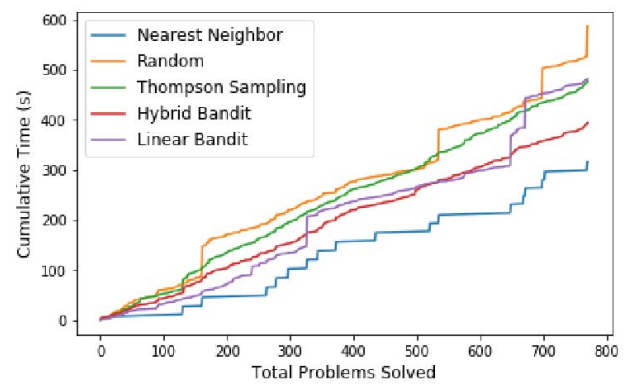

Figure 2: A nearest neighbor algorithm appears to be faster than most other state of the art algorithm selection approaches.

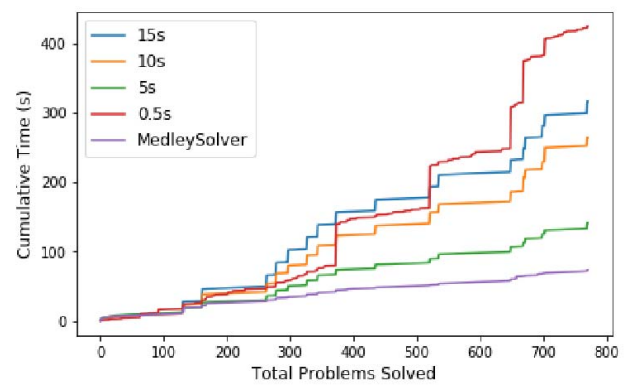

Figure 3: Through exponential estimation, MedleySolver is able to significantly reduce timeouts safely, leading to a reduced runtime.

Solver improves upon the performance of all inputted solvers, and can deliver results far faster than offline learning systems.

In Fig. 2, we see the superiority of nearest neighbor to several other approaches to the contextual bandit problem, including Thompson sampling [1], linear contextual models [6], and random ordering. All algorithms had constant timeouts. This answers our third research question; the nearest neighbor approach triumphs.

In Fig. 3, we can see how our exponential estimation scheme makes the solver faster. All lines use nearest-neighbor classification. Lower timeouts lead to a faster overall time up to a certain point, but if timeouts are too small, solvers are cut off before they can complete the problem This answers our fourth research question: exponential estimation allows the solver to reduce timeouts without running into the issues associated with an overly short cutoff.

\section{CONCLUSIONS}

We have developed an online learning algorithm for SMT solver selection and empirically proven that it provides significant speedup over any individual SMT solver when evaluating a dataset of queries. Our results show MedleySolver makes nearly optimal solver selection without costly offline training. In fact, MedleySolver can solve roughly 1.25 million queries before tools like FastSMT have even finished training: our tool provides speedup out of the box without any delay. In the future, we intend to evaluate our tool in different SMT domains, experiment with additional featurization, and enable generation of an interpretable classifier after sufficient training. 


\section{ACKNOWLEDGMENTS}

Thank you to Federico Mora, Elizabeth Polgreen, and Sanjit A. Seshia for mentoring me.

\section{REFERENCES}

[1] Shipra Agrawal and Navin Goyal. 2012. Analysis of Thompson Sampling for the Multi-armed Bandit Problem. In Proceedings of the 25th Annual Conference on Learning Theory (Proceedings of Machine Learning Research), Shie Mannor, Nathan Srebro, and Robert C. Williamson (Eds.), Vol. 23. PMLR, Edinburgh, Scotland, 39.1-39.26. http://proceedings.mlr.press/v23/agrawal12.html

[2] Mislav Balunovic, Pavol Bielik, and Martin Vechev. 2018. Learning to Solve SMT Formulas. In Advances in Neural Information Processing Systems 31, S. Bengio, H. Wallach, H. Larochelle, K. Grauman, N. Cesa-Bianchi, and R. Garnett (Eds.). Curran Associates, Inc., 10337-10348. http://papers.nips.cc/paper/8233-learningto-solve-smt-formulas.pdf

[3] Clark Barrett, Christopher L. Conway, Morgan Deters, Liana Hadarean, Dejan Jovanovi'c, Tim King, Andrew Reynolds, and Cesare Tinelli. 2011. CVC4. In Proceedings of the 23rd International Conference on Computer Aided Verification (CAV '11) (Lecture Notes in Computer Science), Ganesh Gopalakrishnan and Shaz Qadeer (Eds.), Vol. 6806. Springer, 171-177. Snowbird, Utah.

[4] Leonardo De Moura and Nikolaj Bjørner. 2008. Z3: An Efficient SMT Solver. In Proc. of TACAS'08. Springer, 337-340.
[5] Bruno Dutertre. 2014. Yices 2.2. In Computer-Aided Verification (CAV'2014) (Lecture Notes in Computer Science), Armin Biere and Roderick Bloem (Eds.), Vol. 8559. Springer, 737-744.

[6] Lihong Li, Wei Chu, John Langford, and Robert E. Schapire. 2010. A ContextualBandit Approach to Personalized News Article Recommendation. In Proceedings of the 19th International Conference on World Wide Web (WWW'10). Association for Computing Machinery, New York, NY, USA, 661-670. https://doi.org/10. $1145 / 1772690.1772758$

[7] In Jae Myung. 2003. Tutorial on Maximum Likelihood Estimation. 7. Math. Psychol. 47, 1 (Feb. 2003), 90-100. https://doi.org/10.1016/S0022-2496(02)00028-7

[8] Aina Niemetz, Mathias Preiner, and Armin Biere. 2019. Boolector at the SMT competition 2019. In Proceedings of the 17th International Workshop on Satisfiability Modulo Theories (SMT 2019), affiliated with the 22nd International Conference on Theory and Applications of Satisfiability Testing (SAT 2019), Lisbon, Portugal, fuly 7-8, 2019, Joe Hendrix and Natasha Sharygina (Eds.). 2 pages.

[9] Nikhil Pimpalkhare. 2020. MedleySolver. https://github.com/nikhilpim/ medleysolver.

[10] Preiner, Mathias, Niemetz, Aina, Scott, Joseph, and Ganesh, Vijay. 2020. MachSMT: A Machine Learning-based Algorithm Selector for SMT Solvers. (2020). http: //hdl.handle.net/10012/15755

[11] Tjark Weber, Sylvain Conchon, David Déharbe, Matthias Heizmann, Aina Niemetz, and Giles Reger. 2019. The SMT Competition 2015-2018. 7. Satisf. Boolean Model. Comput. 11, 1 (2019), 221-259. https://doi.org/10.3233/SAT190123 